\title{
SKLB-287, a novel oral multikinase inhibitor of EGFR and VEGFR2, exhibits potent antitumor activity in LoVo colorectal tumor model
}

X. CHEN ${ }^{1}$, Y. LIU², H.-W. YANG ${ }^{1}$, S. ZHOU ${ }^{1}$, C. CHENG ${ }^{1}$, M.-W. ZHENG ${ }^{1}$, L. ZHONG ${ }^{1}$, X.-Y. FU ${ }^{3}$, Y.-L. PAN ${ }^{1}$, S. MA ${ }^{1}$, Y. TANG ${ }^{3}$, Y.-Z. CHEN ${ }^{1}$, L.-L. LI ${ }^{3, *}$, S.-Y. YANG ${ }^{1, *}$

${ }^{1}$ State Key Laboratory of Biotherapy and Cancer Center, West China Hospital, West China Medical School, Sichuan University, Chengdu, Sichuan 610041, China; ${ }^{2}$ Department of Urology, the 452nd Hospital of People's Liberation Army, Chengdu 610021, China; ${ }^{3}$ West China School of pharmacy, Sichuan University, Sichuan 610041, China

*Correspondence: yangsy@scu.edu.cn,ysylilinli@sina.com

Received July 22, 2013 / Accepted January 28, 2014

\begin{abstract}
Colorectal cancer (CRC) is the third common cancer and most of the chemotherapies of CRC currently used often suffer limited efficacy and large side effects. Targeted small-molecule by anti-tumor drugs are thought a promising strategy for improving the efficacy and reducing the side effects. In this investigation, we report a novel multikinase inhibitor, termed SKLB-287, which was discovered by us recently. SKLB-287 could efficiently inhibit the activation of endothelial growth factor receptor (EGFR) and vascular endothelial growth factor receptor 2 (VEGFR2). It displayed very good anti-proliferative activity against LoVo CRC cells and considerable antiangiogenic potency in transgenic zebrafish embryos. Oral administration of SKLB-287 resulted in dose-dependent suppression of tumor growth in LoVo xenograft mouse model. Immunohistochemistry was adopted to examine the in vivo anti-tumor mechanism of action of SKLB-287.
\end{abstract}

Key words: SKLB-287, EGFR, antiangiogenesis, colorectal cancer

Colorectal cancer (CRC) is the third most common cancer worldwide[1]. Although the morbidity and mortality of colorectal cancer have declined in the past decade, 51,590 patients died of colorectal cancer in 2012 in the United States, accounting for $9 \%$ of all cancer-related deaths[2]. Surgical resection is an effective curative method for early-stage CRC. However, approximately $60 \%$ of patients with metastatic and advanced $\mathrm{CRC}$ are diagnosed during the late stages of the disease and are not eligible for surgery. Chemotherapy and radiotherapy are the only therapeutic options for these patients. Unfortunately, most of the chemotherapies currently used show poor efficacy and bear large side effects [3]. Targeted small-molecule antitumor drugs are thought a promising strategy for improving the efficacy and reducing the side effects [4].

The epidermal growth factor receptor is a member of the ErbB family of receptors. Numerous studies have demonstrated that aberrant expression or mutation of EGFR could result in various cancers [5]. Indeed, increased EGFR expression is observed in majority of CRC $(>70 \%)$ cases, which has been shown to associate with the development of CRC and poor prognosis[6-9]. Thus, EGFR has been consid- ered as a potential target for the treatment of CRC $[10,11]$. Several studies have indicated that EGFR inhibitor gefitinib in combination with standard chemotherapy had a curative effect in a fraction of CRC patients[12,13]. Furthermore, two EGFR-targeted monoclonal antibodies, cetuximab[14-17] and panitumumab[18-21], have been approved by the United States Food and Drug Administration (US FDA) for the treatment of advanced CRC[22].

Uncontrolled angiogenesis is a typical characteristic of most human solid tumors, including CRC. Inhibition of angiogenesis is also thought a potential approach for the treatment of CRC $[23,24]$. Of all the known angiogenic molecules, VEGF is the key mediator promoting angiogenesis. VEGF and its receptors are thus considered as important targets for antiangiogenetic therapy in cancer. Bevacizumab is a VEGF targeting monoclonal antibody, which was approved for the treatment of advanced CRC by US FDA in 2004[22,25,26]. Small molecule VEGFR inhibitors, such as axitinib, also showed considerable efficacy in the treatment of CRC.[27]

It is reasonable to hypothesize that a multi-targeted agent that can simultaneously inhibit EGFR signaling and suppress 
tumor angiogenesis might have enhanced therapeutic efficacy and benefit reducing the risk of drug resistance. SKLB-287 (N8-(4-bromo-2-fluorophenyl)-9-cyclopentyl-N2-(4-(4methylpiperazin-1-yl)phenyl)-9H-purine-2,8-diamine, Fig. 1 ) is such a small molecule multikinase inhibitor that can efficiently inhibit EGFR and VEGFR2, which was discovered by us recently[28]. In this investigation, we shall assess the anti-CRC activities of SKLB-287 both in vitro and in vivo.

\section{Materials and methods}

Cell lines. Human colorectal cancer cell line LoVo, SW480, HCT116, and SW620, human lung cancer cell line H1299, human breast cancer cell line MCF-7, human hepatocarcinoma cell line PLC/PRF/5, and HepG2, and human melanoma cell line A2058, and C32, were obtained from American Type Culture Collection (ATCC, Rockville, MD, USA). Human hepatocarcinoma cell line BEL7402, and SMMC7721were obtained from the Cell Culture Center of the Institute of Basic Medical Sciences of Chinese Academy of Medical Sciences and School of Basic Medicine of Peking Union Medical College (Beijing, China). All of them were grown in DMEM or RPMI1640 medium supplemented with 10\% Fetal Bovine Serum (FBS) (Gibco, Eggenstein, Germany), 100 units/ml penicillin (Sigma-Aldrich) and streptomycin (Sigma-Aldrich), and maintained in the $37^{\circ} \mathrm{C}$ incubator with a humidified $5 \%$ $\mathrm{CO}_{2}$.

Preparation of SKLB-287. SKLB-287 was synthesized in the State Key Laboratory of Biotherapy, Sichuan University (Sichuan, China). Gefitinib was obtained from commercial sources. The chemical structure of SKLB-287 was presented in Fig. 1. For all in vitro assays and zebrafish assays, SKLB-287 was prepared initially as a $10 \mathrm{mM}$ stock solution in dimethyl sulfoxide (DMSO). Stock solution of SKLB-287 was diluted in optimal assay buffers or culture media with $0.1 \%$ DMSO served as a vehicle control. For in vivo studies, SKLB-287 was dissolved in aqueous DMSO-PEG400 solution, with $4 \%(\mathrm{v} / \mathrm{v})$ DMSO (Sigma, Saint Louis, MO, USA), 25\%(v/v) PEG400 (Sigma, Saint Louis, MO, USA), and 71\%(v/v) $\mathrm{H}_{2} \mathrm{O}$, and administered at doses of $12.5,25$ and $50 \mathrm{mg} / \mathrm{kg}$, respectively. Gefitinib was dissolved in the same solvent at a dose of $100 \mathrm{mg} / \mathrm{kg}$.

Kinase inhibition assays. Kinase inhibitory activities of SKLB-287 were tested by radiometric assays provided by Kinase Profiler Service (Millipore, Billerica, MA USA) as previously described [29].

Cell proliferation assays. Anti-proliferative activities of SKLB-287 were measured using 3-(4,5)-dimethylthiahiazo($\mathrm{z}-\mathrm{y1}$ )-3,5-di-phenytetrazoliumromide (MTT) assays and EdU incorporation assays. Various cells $\left(2 \times 10^{3}\right.$ to $8 \times 10^{3}$ cells/well $)$ were treated with indicated concentrations of SKLB-287 for $72 \mathrm{~h}$. Each assay was repeated 3 times. The $\mathrm{IC}_{50}$ values were calculated by GraphPad Prism 5.01 software (Prism Statistical Software). LoVo cells were seeded in 96-well plate at a density of $8 \times 10^{3}$ per well. Then, the proliferative cells were assayed

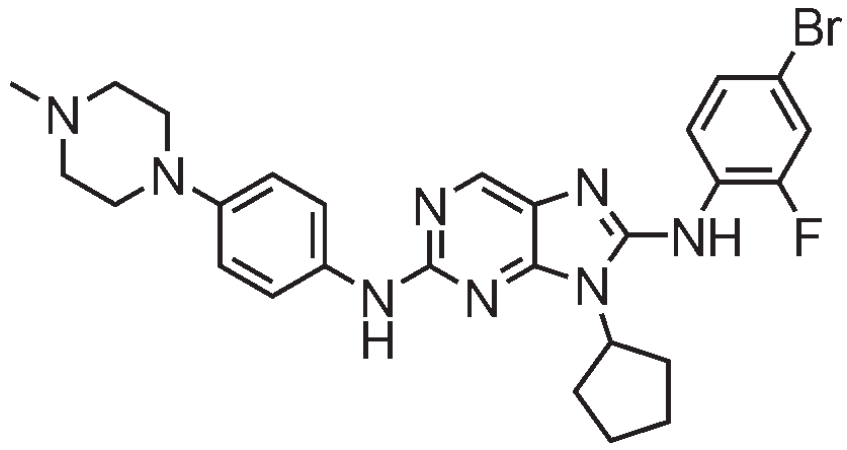

Figure 1. The chemical structure of SKLB-287.

with an EdU-Apollo ${ }^{567}$ DNA Proliferation Detection kit according to the manufacturer's instructions. Images were taken by High Content Screening and Analysis (Thermo Fisher Cellomics).

Apoptosis analysis by flow cytometry (FCM). FCM assays were done to identify apoptotic cells with or without SKLB-287 treated and to analysis the percentage of apoptotic cells after propidium iodide (PI) staining in hypotonic buffer. Cells were collected and washed with cold PBS. Then $0.4 \mathrm{ml}$ hypotonic fluorochrome solution containing $50 \mu \mathrm{g} / \mathrm{ml} \mathrm{PI}$ in $0.1 \%$ sodium citrate plus $0.1 \%$ Triton X-100 was added to cells and 10000 cells were analyzed by FCM (ESP Elite, Beckman-Coulter, Miami, FL, USA). Apoptotic cells appeared in the cell cycle distribution as cells with a DNA content of less than that of G1 cells and were estimated with Listmode software.

Western blot analysis. In assays for examining the mechanisms of action of SKLB-287 on the LoVo cells, LoVo cells were incubated with SKLB-287 for $8 \mathrm{~h}$. Cells were lysed with ripa buffer (Biotime) containing 0.1\% PMSF and 1\% proteinase inhibitor cocktail (Sigma). Protein content was determined using Bio-Rad Protein Assay Kit (Bio-Rad). Lysates were mixed with $5 \times$ sodium dodecyl sulfate (SDS)- polyacrylamide gel electrophoresis (PAGE) sample loading buffer (Beyotime) and denatured in a boiling water bath for 3-5 min and equalized before loading. Protein samples were separated by $10 \%$ SDSPAGE gels and transferred onto a polyvinylidene difluoride (PVDF) membrane (Millipore). Membranes were blocked in blocking buffer (Tris-buffered saline (TBS) containing 0.1\% Tween 20 and 5\% nonfat dry milk) for $1 \mathrm{~h}$. All antibodies were diluted in blocking buffer. Blots were incubated with the corresponding primary antibody $(1: 1000)$ such as anti-EGFR, anti-p-EGFR, anti-AKT, anti-p-AKT, anti-P38, anti-p-P38, anti-MET, anti-p-MET, anti-ERK, anti-p-ERK, anti-SRC, and anti-p-SRC (Cell Signaling Technology), and anti-GAPDH (Santa Cruz) overnight. Then, blots were incubated for $1 \mathrm{~h}$ with the corresponding horseradish peroxidase-linked secondary antibodies (Santa Cruz) diluted 1:5000 in the same buffer. Following secondary antibody reaction, the proteins were detected with BeyoECL reagents (Millipore) by exposure on a Kodak film. 
In vivo antiangiogenesis assays in transgenic zebrafish. The FLK-1 promoter EGFP transgenic zebrafish (FLK-1: EGFP) was used to investigate the in vivo antiangiogenic activity of SKLB-287 as previously described [30]. 10 embryos per experimental group were used in our study, and each experiment was performed in 3 replicates. Embryos placed in 24-well plates were maintained in Holtfreter's solution in a humidified incubator at $28.5^{\circ} \mathrm{C}$. Zebrafish embryos were incubated overnight with $1.25,2.5$ and $5 \mu \mathrm{M}$ SKLB-287 or vehicle from $12 \mathrm{~h}$ post-fertilization (hpf) until $33 \mathrm{hpf}$. At $33 \mathrm{hpf}$, zebrafish were anesthetized with $0.01 \%$ tricaine and imaged under the fluorescence microscope (Carl Zeiss Microimaging Inc.) equipped with an AxioCam MRc5 digital CCD camera (Carl Zeiss Microimaging Inc.).

Pharmacodynamics assessment in Xenograft mouse models. Animal studies were conducted in conformity with institutional guide for the care and use of laboratory animals. All mouse protocols were approved by the Animal Care and Use Committee of Sichuan University (Chengdu, Sichuan, China). Six-week-old female athymic mice were obtained from Chinese Academy of Medical Science (Beijing, China). LoVo tumors were established by s.c. injection of $1 \times 10^{7}$ cells. After about two weeks, mice bearing tumors around 150-200 $\mathrm{mm}^{3}$ were selected and randomized into treatment groups (6 mice per group). The animals were orally gavaged daily with SKLB-287, Gefitinib, and vehicle control. Tumor length and width were measured every three days, and tumor volume (TV) was calculated using the following expression: $\mathrm{TV}=$ length $\times$ width $^{2} \times 0.5$. At the end of experiment, mice were sacrificed.

Immunohistochemistry. Solid tumors were removed and processed to immunohistochemical analysis when the mice were administrated after 12 days, half of the tumors were fixed in formalin followed by paraffin-embedded and the other were stored at $-80^{\circ} \mathrm{C}$. To investigate the tumor proliferation inhibition potencies of SKLB-287, we examined the proliferous cells by immunostaining with Ki67 (Thermo Fisher Scientific), apoptosis cells by TUNEL (Promega Corporation), p-EGFR (Cell Signaling Technology) and p-ERK (Cell Signaling Technology) for MAPK signaling pathway in paraffin-embedded tumors as described previously[31]. To investigate whether SKLB-287 inhibited tumor growth by suppressing tumor angiogenesis, we examined the vessel density in tumor tissue as described previously [32]. To determine vessel density, CD31 (BD Biosciences) were examined in frozen sections of LoVo

Table 1. Kinase inhibition profile for SKLB-287 against a panel of selected protein kinases.

\begin{tabular}{lccc}
\hline Kinase & $\mathrm{IC}_{50}(\mu \mathrm{M})$ & Kinase & Inhibitory activity @ $10 \mu \mathrm{M}(\%)$ \\
\hline EGFR & 0.061 & SRC & 100 \\
VEGFR2 & 0.088 & MET & 65 \\
& & mTOR & 0 \\
& & Plk1 & 0 \\
\hline
\end{tabular}

tumor xenografts and p-VEGFR2 (Cell Signaling Technology) in paraffin-embedded tumors.

Statistical analysis. The data are reported as the mean \pm SD. Student's $t$-test was used to analyze differences (Prism Statistical Software). Differences were considered statistically significant if $P<0.05$.

\section{Results}

Kinase inhibition profile of SKLB-287. The kinase inhibition profile of SKLB-287 against a panel of recombinant human protein kinases is shown in Table 1 . SKLB-287 potently inhibited EGFR with an $\mathrm{IC}_{50}$ value of $0.061 \mu \mathrm{M}$ and was effective against VEGFR2 with an $\mathrm{IC}_{50}$ value of $0.088 \mu \mathrm{M}$. At a concentration of $10 \mu \mathrm{M}$, SKLB-287 showed $100 \%$ inhibition of SRC activity and 65\% inhibition of MET activity. SKLB287 displayed almost no inhibitory activity against mTOR and plk1.

In vitro cell growth inhibitory effects of SKLB-287 against various cancer cell lines. The growth inhibitory effects of SKLB-287 against various colorectal cancer cell lines and several other cell lines were measured using MTT assays, and the results are shown in Table 2. SKLB-287 potently inhibited the growth of LoVo cells, which overexpress EGFR, with an $\mathrm{IC}_{50}$ value of $0.456 \mu \mathrm{M}$, showing a potency 6 -fold higher than that of gefitinib. In other colorectal cancer cell lines, including SW480, HCT116, and SW620, SKLB-287 exhibited a weak inhibitory potency, with $\mathrm{IC}_{50}$ values of $2.789 \mu \mathrm{M}, 4.138 \mu \mathrm{M}$, and $6.304 \mu \mathrm{M}$, respectively. SKLB-287 is not sensitive to other EGFR independent cell lines, such as H1299, MCF-7, PLC/ PRF/5, HepG2, A2058, C32, BEL7402, and SMMC7721.

EdU incorporation assays were further conducted to visually assess the antiproliferative activity of SKLB-287. Fig. 2 shows the proliferation of LoVo cells after exposure to SKLB287 at $0.1,0.5$ and $1 \mu \mathrm{M}$. Exposure of LoVo cells to SKLB-287 for $24 \mathrm{~h}$ resulted in a reduction in the number of Hoechst-

Table 2. Cell growth inhibitory potencies of SKLB-287 and gefitinib against various cancer cell lines.

\begin{tabular}{lccl}
\hline Tumor type & $\begin{array}{c}\text { Cancer cell } \\
\text { lines }\end{array}$ & $\begin{array}{c}\mathrm{IC}_{50}(\mu \mathrm{M}) \text { of } \\
\text { SKLB-287 }\end{array}$ & $\begin{array}{c}\mathrm{IC}_{50}(\mu \mathrm{M}) \text { of } \\
\text { gefitinib }\end{array}$ \\
\hline Human colorectal cancer & LoVo & 0.456 & 3.151 \\
Human lung cancer & H1299 & 1.651 & $>10$ \\
Human breast cancer & MCF-7 & 2.613 & $>10$ \\
Human colorectal cancer & SW480 & 2.789 & $>10$ \\
Human hepatocarcinoma & PLC/PRF/5 & 3.682 & $>10$ \\
Human hepatocarcinoma & BEL7402 & 3.694 & $>10$ \\
Human colorectal cancer & HCT116 & 4.138 & $>10$ \\
Human hepatocarcinoma & HepG2 & 4.221 & $>10$ \\
Human melanoma & A2058 & 5.945 & $>10$ \\
Human colorectal cancer & SW620 & 6.304 & $>10$ \\
Human melanoma & C32 & 8.564 & $>10$ \\
Human hepatocarcinoma & SMMC7721 & $>10$ & $>10$ \\
\hline
\end{tabular}




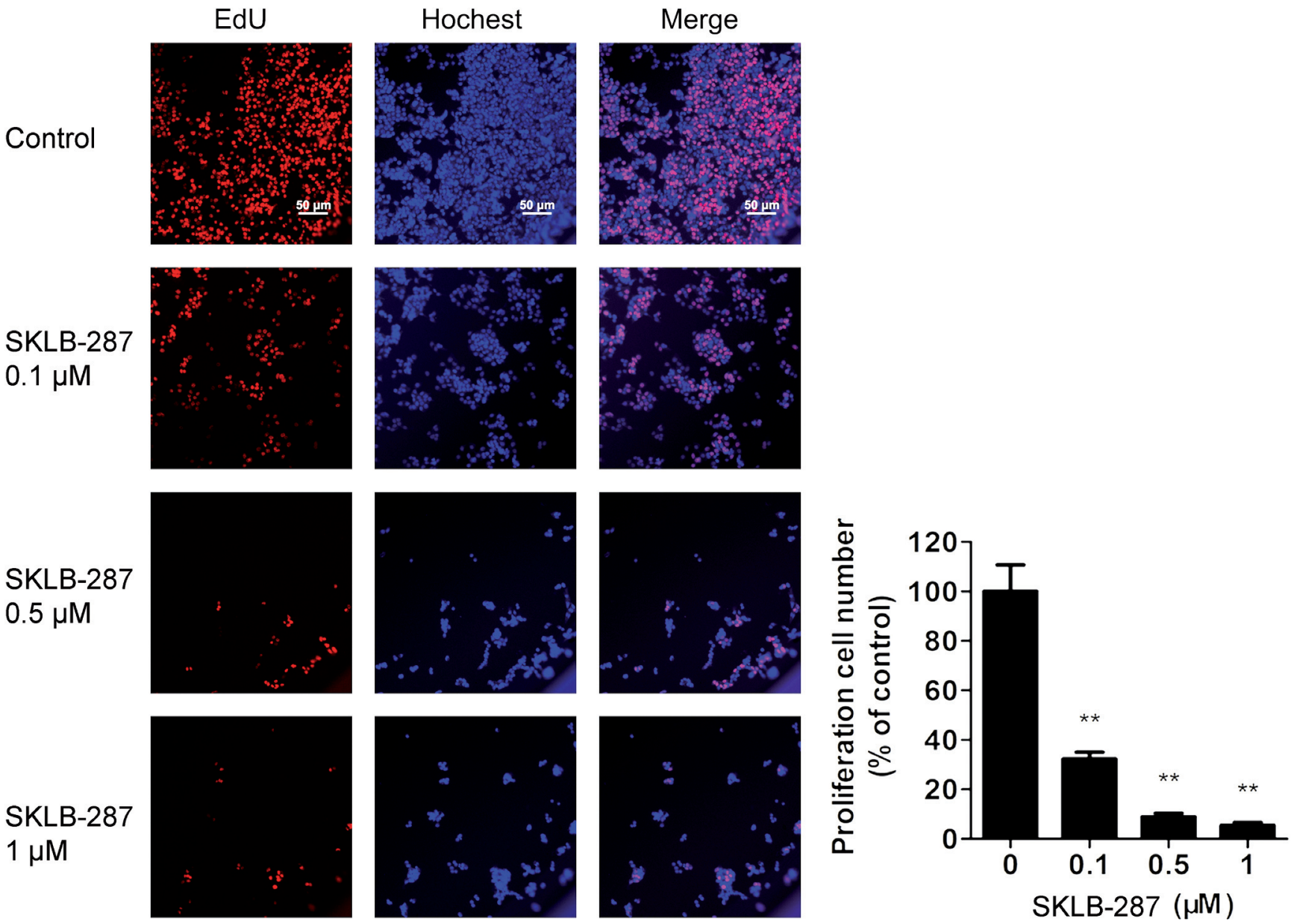

Figure 2. SKLB-287 inhibited LoVo cancer cell proliferation. The proliferating cells were evaluated by EdU kit after a $24 \mathrm{~h}$ SKLB-287 treatment. The blue nuclei and the red represent the nucleus and cells in S phase, respectively. Column, mean; bars, $\mathrm{SD}\left(\mathrm{n}=9\right.$; ${ }^{* *}, \mathrm{P}<0.01$ vs. the control, PRISM).

stained and EdU staining cells, indicating that DNA replication was blocked by treatment with SKLB-287. The results of the EdU incorporation assay also demonstrated that the number of proliferating cells (red nuclei) significantly decreased with increasing concentrations of SKLB-287.

SKLB-287 induced apoptosis in LoVo cells. To determine whether the killing effects of SKLB-287 on LoVo cells were due to apoptosis, LoVo cells were exposed to SKLB-287 at concentrations of $0.1,0.5$, and $1 \mu \mathrm{M}$ for $24 \mathrm{~h}$, and samples showing nuclear propidium iodide staining were analyzed by flow cytometry. Treatment with SKLB-287 at increasing concentrations from 0 to $1 \mu \mathrm{M}$ led to a considerable increase in the number of cells in sub-G1 phase (from $1.01 \%$ to $26.39 \%$ ), indicating that SKLB-287 induced apoptosis in LoVo cells (Fig. 3).

SKLB-287 inhibited the phosphorylation of EGFR and its downstream signaling proteins in LoVo cells. To further examine the mechanisms of action of SKLB-287, its effects on the activity of EGFR and downstream signaling proteins in LoVo cells were assessed by western blotting. As shown in Fig. 4, SKLB-287 inhibited EGFR phosphorylation at $0.1 \mu \mathrm{M}$. SKLB-287 also suppressed the activation of its downstream signaling protein ERK. Furthermore, we examined the phosphorylation status of MET, SRC, AKT (a pro-survival effector), and P38 (a pro-apoptotic effector)[33], which play important roles in cell proliferation and apoptosis, in LoVo cells after exposure to SKLB-287. Our results showed a remarkable decrease in the levels of p-MET, p-SRC, and p-AKT, and an increase in P-P38, whereas the levels of total MET, SRC, AKT, and P38 were unaffected by SKLB-287.

In vivo antiangiogenic effects of SKLB-287. Transgenic zebrafish assays were carried out to examine the antiangiogenic ability of SKLB-287 in vivo. Fig. 5 shows $33 \mathrm{hpf}$ zebrafish embryos treated with $1.25,2.5$, and $5 \mu \mathrm{M}$ SKLB-287, as well as a blank control. Treatment of live fish embryos with SKLB-287 completely blocked the formation of intersegmental vessels (ISVs) at concentrations of $5 \mu \mathrm{M}$ while preserving fluorescence in the dorsal aorta and major 

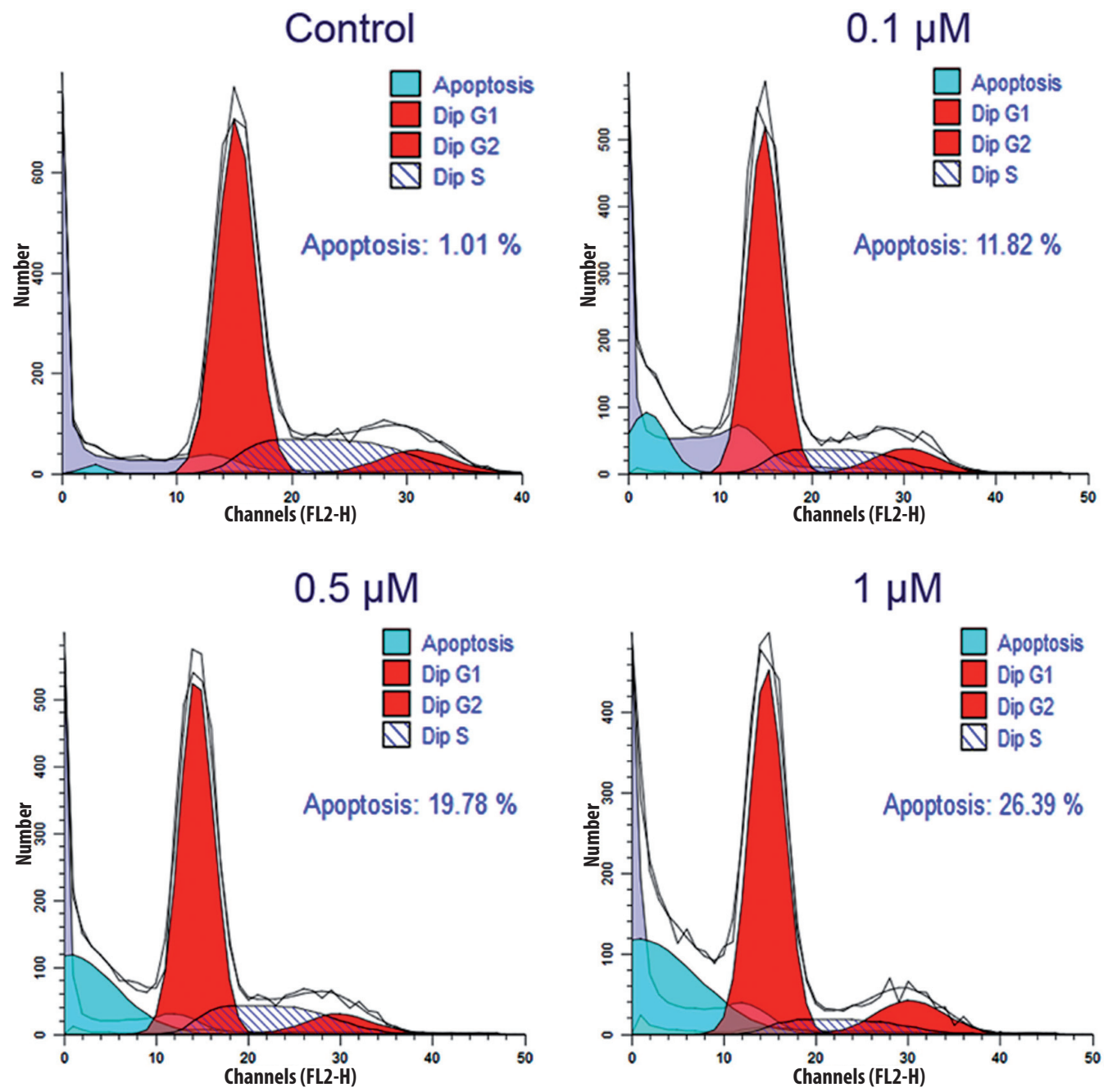

Figure 3. SKLB-287 induced apoptosis in LoVo cells. PI-stained cells were determined by flow cytometry. Treatment various concentrations $(0,0.1,0.5$ and $1 \mu \mathrm{M}$ ) of SKLB-287 for $24 \mathrm{~h}$ corresponded with $1.01 \%, 11.82 \%, 19.78 \%$, and $26.39 \%$ apoptotic cells, respectively. Data were the representative from three parallel experiments.

cranial vessels. At $2.5 \mu \mathrm{M}$ SKLB-287, the formation of intersegmental vessels was considerably inhibited compared with the vehicle control group, indicating a dose-dependent inhibition pattern.

In vivo anti-tumor activity of SKLB-287. The in vivo anti-tumor activity of SKLB-287 was assessed using LoVo xenograft mouse models. When tumors reached a volume of approximately $200 \mathrm{~mm}^{3}$, the mice were grouped and treated orally once daily with $12.5,25$, or $50 \mathrm{mg} / \mathrm{kg}$ per day SKLB-287 for 18 days. The tumor volumes were measured every 3 days. The results showed that $12.5 \mathrm{mg} / \mathrm{kg}$ SKLB-287 could suppress tumor growth with a tumor inhibition rate of approximately $40 \%$ compared with the vehicle group (Fig. 6A). SKLB-287 treatment at $50 \mathrm{mg} / \mathrm{kg}$ per day had considerable tumor-suppressive ability, with a tumor inhibition rate of approximately $60 \%$ compared with the vehicle group, thus showing a higher efficacy than $100 \mathrm{mg} / \mathrm{kg}$ gefitinib. Moreover, during the whole experiment, no significant weight loss or any other obvious signs of toxicity were observed in any of the SKLB-287-treated mice (Fig. 6B). We detected the toxicity of SKLB-287 on the large intestine of normal athymic mice. H\&E and TUNEL staining of paraffin-embedded sections of the large intestine were performed. As shown in Fig. S1A, SKLB-287 did not cause obvious pathologic abnormalities in mouse colon tissues. In addition, SKLB-287 could not induce apoptosis in normal colon cells in vivo (Fig. S1B).

Mechanism of action of SKLB-287 in the LoVo tumor xenograft model. Immunohistochemical analyses were carried out to examine mechanism of action of SKLB-287 in the LoVo tumor xenograft model. To investigate the tumor proliferation inhibition potencies of SKLB-287, we examined the proliferous cells by immunostaining with Ki67. We found that there were 
fewer Ki67-positive cells in SKLB-287-treated group, implying a reduction in the number of proliferating cells (Fig. 7A). Then TUNEL staining was performed to analyze the effect of SKLB287 on apoptosis in the LoVo xenograft tumors. As shown in Fig. 7B, tumors treated with $25 \mathrm{mg} / \mathrm{kg}$ SKLB-287 had a higher number of TUNEL-positive cells with dark green fluorescent staining, indicating that SKLB-287 induced apoptosis in LoVo cells. Considering that MAPK families play an important role in regulating cell proliferation and survival[34], we subsequently detected the phosphorylation of EGFR and ERK (Fig. 7C). Compared with the vehicle-treated control group, the $25 \mathrm{mg} / \mathrm{kg}$ SKLB-287-treated group showed a significant decrease in the number of p-EGFR and p-ERK positive cells. To investigate whether SKLB-287 inhibited tumor growth by suppressing tumor angiogenesis, we examined the vessel density in tumor tissue with CD31 and p-VEGFR2. The results showed that SKLB-287 considerably reduced tumor microvessel density in LoVo xenografts, as assessed by CD31 staining (Fig. 7A). As shown in Fig. 7C, tumors treated with $25 \mathrm{mg} / \mathrm{kg} \mathrm{SKLB}-287$ had a reduced number of p-VEGFR2-positive cells.

\section{Discussion}

Although several monoclonal antibodies have been approved by US FDA for the treatment of advanced CRC, there is no small molecule targeted drug approved for clinical treatment of CRC $[14,15,20]$. Thus, there is a significant medical need for development of such targeted small molecule drugs for the therapy of CRC.

Relative to single target agents, multi-target drugs are thought to be superior in treating complex diseases like CRC in terms of efficacy and ability of overcoming drug-resistance. Furthermore, it has been reported that combination therapy with agents targeting the EGFR and VEGF pathways could significantly enhance anti-tumor efficacy compared with sole use of these agents in CRCs[35,36]. SKLB-287 is such a multi-tar-

$\begin{array}{lllll}\begin{array}{l}\text { SKLB-287 } \\ (\mu \mathrm{M})\end{array} & 0 & 0.1 & 0.5 & 1\end{array}$

p-EGFR

EGFR

p-AKT

AKT

$p-E K R$

ERK

p-MET

MET

p-SRC

SRC

p-P38

P38
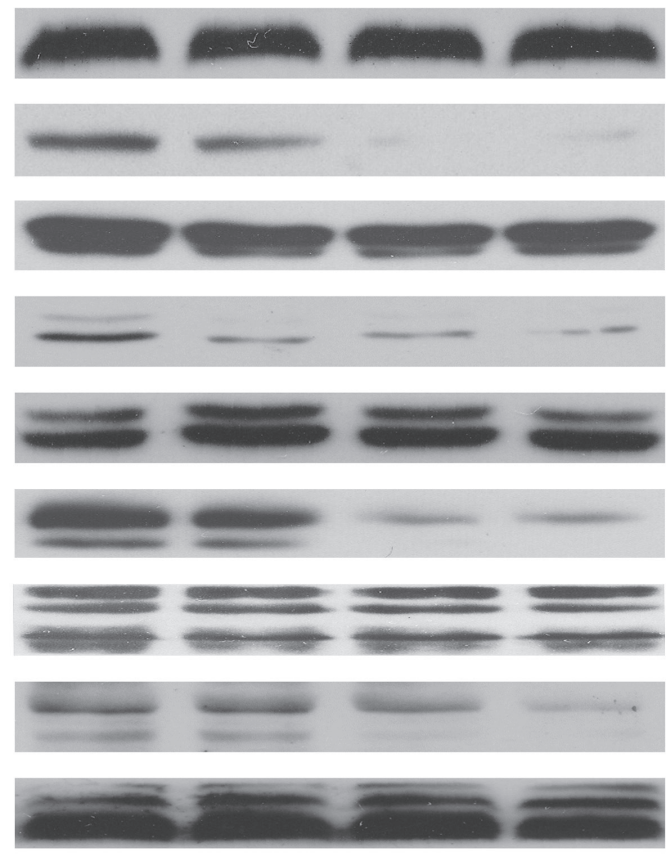

Figure 4. Inhibition of EGFR signaling pathway of LoVo cells. LoVo cells were exposed to $0,0.1,0.5$, and $1 \mu \mathrm{M}$ SKLB-287 for $8 \mathrm{~h}$ and cell extract proteins subjected to Western blotting analysis.

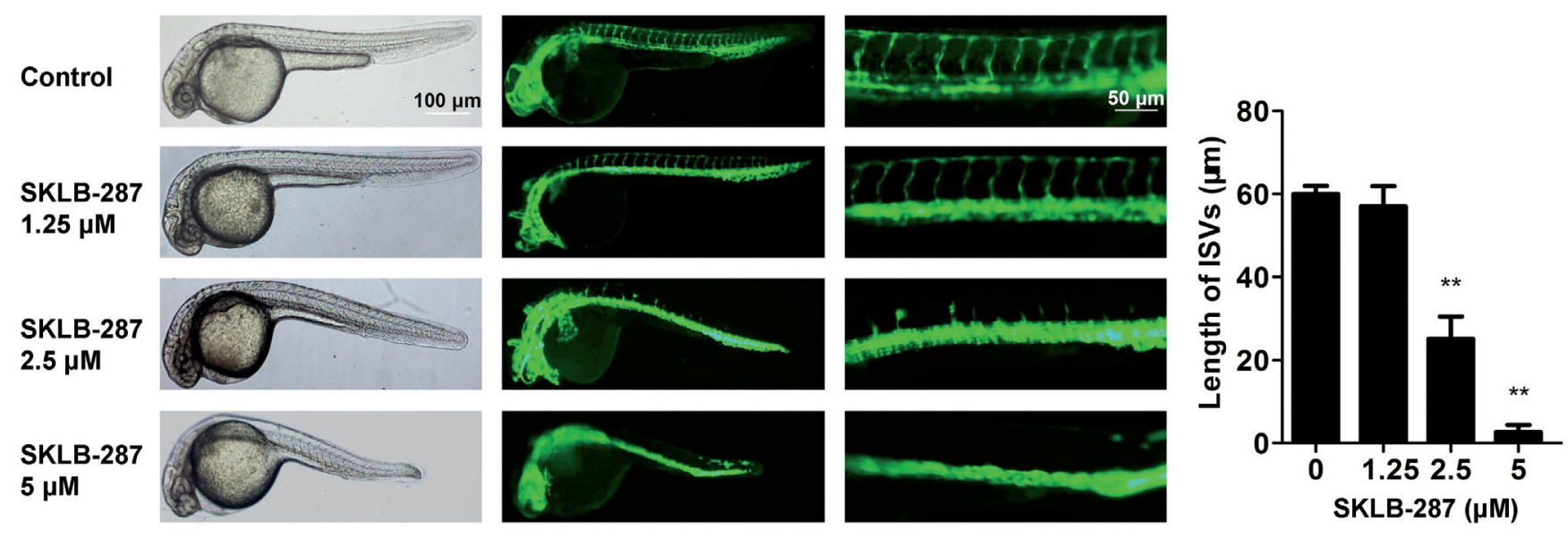

Figure 5. SKLB-287 inhibits angiogenesis in vivo. $33 \mathrm{hpf}$ zebrafish embryos treated with 1.25, 2.5 and $5 \mu \mathrm{M}$ SKLB-287. ISV budding and outgrowth were almost completely inhibited by treatment with $5 \mu \mathrm{M}$ SKLB-287. Column, mean; bars, $\mathrm{SD}\left(\mathbf{n}=10\right.$; ${ }^{* *}, \mathrm{P}<0.01$ vs. the control, PRISM). 
(A)

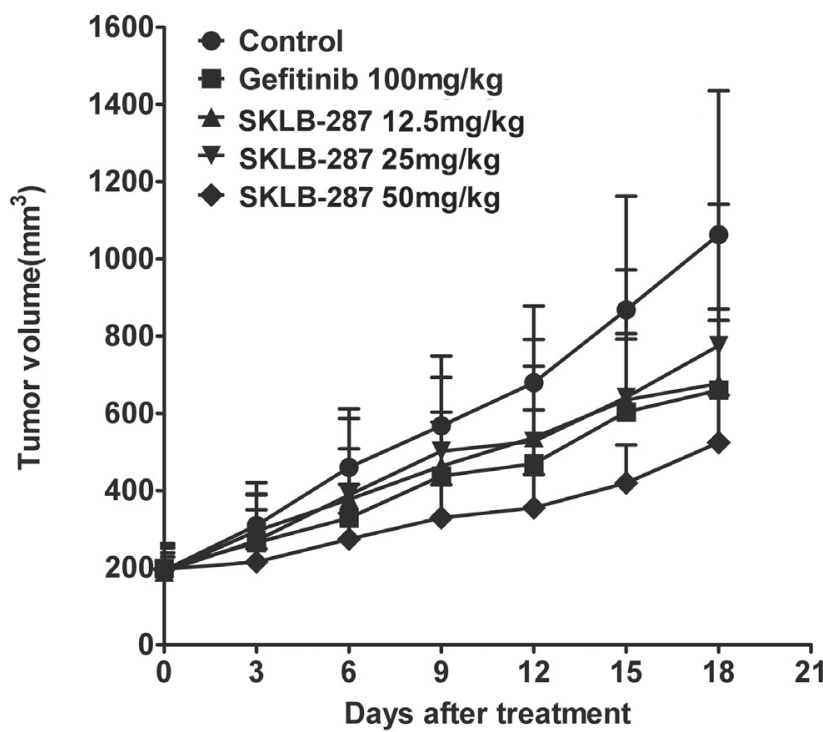

(B)

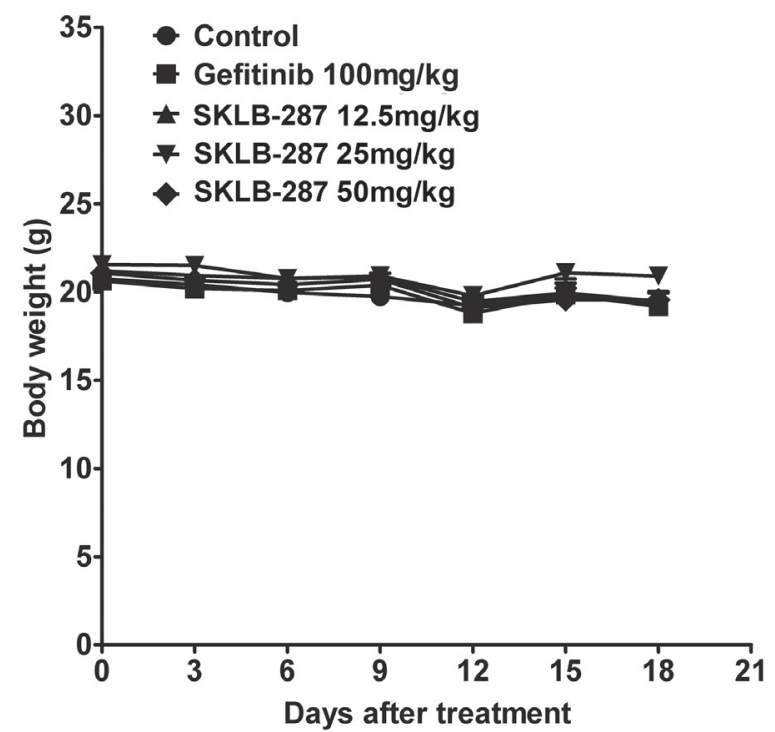

Figure 6. SKLB-287 inhibits tumor growth in LoVo xenograft models. (A) Mice implanted with LoVo xenografts were treated when the tumors grew to about $200 \mathrm{~mm}^{3}$. Animals (6 per group) were treated with vehicle, SKLB-287 at doses of 12.5, 25, and 50 mg/kg, or gefitinib at a dose of $100 \mathrm{mg} / \mathrm{kg}$ once daily for $18 \mathrm{~d}$. Efficacy data are plotted as mean tumor volume (in $\left.\mathbf{m m}^{3}\right) \pm \mathrm{SD}$; $(\mathrm{n}=6)$. (B) Body weight of animals in LoVo xenograft models. Mean body weight $(g) \pm S D$; $(n=6)$.

(A)

Ki67

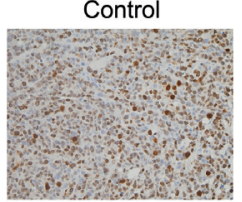

CD31

(B)

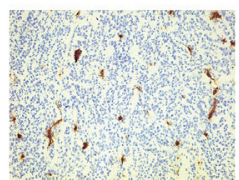

DAPI
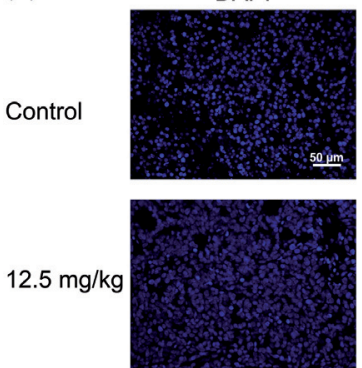

$25 \mathrm{mg} / \mathrm{kg}$

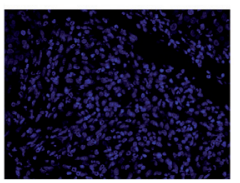

$12.5 \mathrm{mg} / \mathrm{kg}$
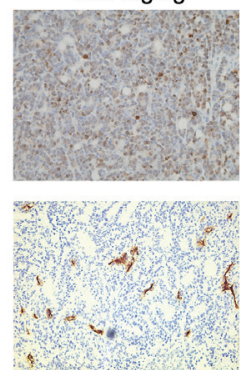

TUNEL
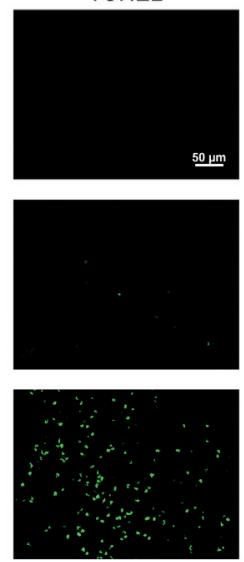

$25 \mathrm{mg} / \mathrm{kg}$
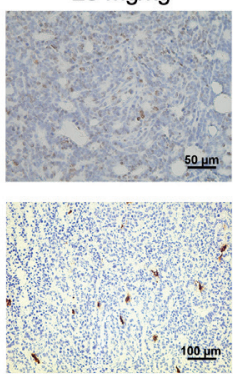

Merge

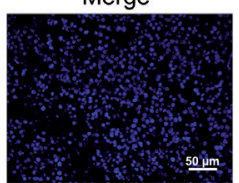

$\mathrm{p}-\mathrm{VEGFR2}$

(C)

p-EGFR

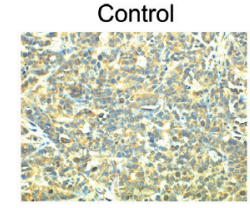

p-ERK
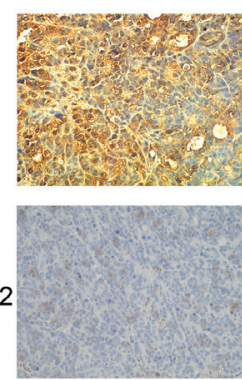

$12.5 \mathrm{mg} / \mathrm{kg}$
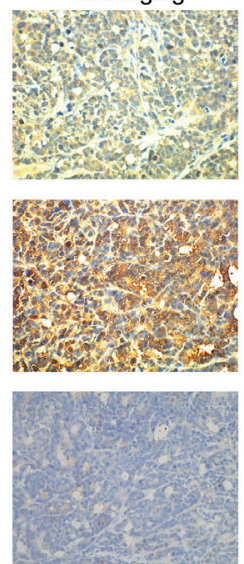

$25 \mathrm{mg} / \mathrm{kg}$
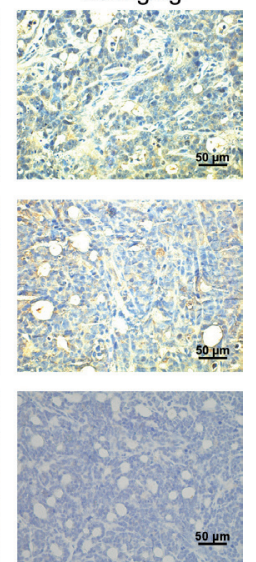
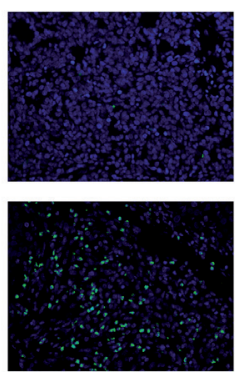

Figure 7. Mechanism of action of SKLB-287 in LoVo tumor xenograft models. Animals bearing LoVo tumor xenograft were treated with SKLB-287 at 12.5 , and $25 \mathrm{mg} / \mathrm{kg}$, or vehicle once a day for 12 days. After 12 days of SKLB-287 treatment, the LoVo tumors (3 per group) were collected separately.

(A) Histological sections of each group tumors were stained with Ki67 and CD31. (B) Apoptosis in LoVo cells in vivo were detected by TUNEL assays.

(C) Histological sections were stained with p-EGFR, p-VEGFR2, and p-ERK. 
get inhibitor that potently inhibits EGFR and VEGF signaling. In enzymatic assays, SKLB-287 showed a good potency against both EGFR ( IC $\left._{50}: 0.061 \mu \mathrm{M}\right)$ and VEGFR2 (IC $\left.50.008 \mu \mathrm{M}\right)$. In cell assays, SKLB-287 exhibited a higher anti-proliferative activity against LoVo colorectal cancer cells than gefitinib did, with an $\mathrm{IC}_{50}$ value of $0.456 \mu \mathrm{M}$ for SKLB-287 and $3.151 \mu \mathrm{M}$ for gefitinib. It also showed greater in vivo anti-tumor activity in LoVo xenograft mouse models than gefitinib with less dosing (50 mg/kg SKLB-287 vs $100 \mathrm{mg} / \mathrm{kg}$ gefitinib).

In studies of mechanism of action, SKLB-287 displayed considerable potency in blocking EGFR signaling in intact LoVo cells. It also down-regulated p-AKT and up-regulated pP38, which could be used to explain the pro-apoptotic effect of SKLB-287[33]. Results from immunohistochemical analyses indicated that the percentage of Ki67-expressing cells in tumor tissues was considerably lower following SKLB-287 treatment, indicating a reduction in the number of proliferating cells in the tumors[37]. Furthermore, it was shown that SKLB-287 decreased the number of p-VEGFR2 positive cells, and the microvessel density compared with vehicle-treated tumors[38]. Overall, SKLB-287 plays its anti-tumor roles mainly through anti-proliferative effect and anti-angiogenesis. However, it is difficult to exactly differentiate how much of the observed in vivo effects of SKLB-287 are due to its tumor cell suppression and how much due to anti-angiogenic effects.

In summary, we examined the anti-colorectal cancer activities of the novel multi-kinase inhibitor SKLB-287 both in vitro and in vivo. SKLB-287 exerts its anti-tumor activity mainly through the inhibition of MAPK signaling and its antiangiogenic effect. In this model, SKLB-287 was more effective than gefitinib, presumably due to additional target compared to gefitinib. SKLB-287 has the convenience of oral administration and low toxicity. Summarizing, this study establishes a favorable preclinical profile of SKLB-287, which may support SKLB-287 as a promising candidate for clinical studies as a single agent or in combination with other targeted agents or chemotherapeutic drugs in patients with colorectal cancer.

Supplementary information is available in the online version of the paper.

Acknowledgements: This work was supported by National Basic Research Program of China (973 Program) (2013CB967204), National Science and Technology Major Project (2012ZX09501001-003), the National Natural Science Foundation of China (81172987), and PCSIRT (IRT13031).

\section{References}

[1] SHAW P, COSTA J. Molecular biology of colon cancer (review). Anticancer Res 1989; 9: 21-27.

[2] SOCIETY AC CANCER FACTS \& FIGURES 2012. Atlanta: American Cancer Society 2012.

[3] SAIF MW. Targeted agents for adjuvant therapy of colon cancer. Clin Colorectal Cancer 2006; 6: 46-51. http://dx.doi. org/10.3816/CCC.2006.n.020
[4] EL ZOUHAIRI M, CHARABATY A, PISHVAIAN MJ. Molecularly targeted therapy for metastatic colon cancer: proven treatments and promising new agents. Gastrointest Cancer Res 2011; 4: 15-21.

[5] HYNES NE, LANE HA. ERBB receptors and cancer: the complexity of targeted inhibitors. Nat Rev Cancer 2005; 5: 341-354. http://dx.doi.org/10.1038/nrc1609

[6] KRASINSKAS AM. EGFR Signaling in Colorectal Carcinoma. Patholog Res Int 2011; 2011: 932932.

[7] VAN SCHAEYBROECK S, KARAISKOU-MCCAUL A, KELLY D, LONGLEY D, GALLIGAN L, et al. Epidermal growth factor receptor activity determines response of colorectal cancer cells to gefitinib alone and in combination with chemotherapy. Clin Cancer Res 2005; 11: 7480-7489. http://dx.doi.org/10.1158/1078-0432.CCR-05-0328

[8] SPANO JP, FAGARD R, SORIA JC, RIXE O, KHAYAT D, et al. Epidermal growth factor receptor signaling in colorectal cancer: preclinical data and therapeutic perspectives. Ann Oncol 2005; 16: 189-194. http://dx.doi.org/10.1093/annonc/ $\underline{\operatorname{mi} 057}$

[9] MCKAY JA, MURRAY LJ, CURRAN S, ROSS VG, CLARK $\mathrm{C}$, et al. Evaluation of the epidermal growth factor receptor (EGFR) in colorectal tumours and lymph node metastases. Eur J Cancer 2002; 38: 2258-2264. http://dx.doi.org/10.1016/ S0959-8049(02)00234-4

[10] JIMENO A, GRAVALOS C, ESCUDERO P, SEVILLA I, VEGA-VILLEGAS ME, et al. Phase I/II study of gefitinib and capecitabine in patients with colorectal cancer. Clin Transl Oncol 2008; 10: 52-57. http://dx.doi.org/10.1007/s12094-0080153-5

[11] PEREZ-SOLER R. The role of erlotinib (Tarceva, OSI 774) in the treatment of non-small cell lung cancer. Clin Cancer Res 2004; 10: 4238s-4240s. http://dx.doi.org/10.1158/1078-0432. CCR-040017

[12] TRARBACH T, REINACHER-SCHICK A, HEGEWISCHBECKER S, VANHOEFER U, FRIELING T, et al. Gefitinib in combination with capecitabine as second-line therapy in patients with advanced colorectal cancer (aCRC): a phase I/II study of the Arbeitsgemeinschaft Internistische Onkologie (AIO). Onkologie 2010; 33: 89-93. http://dx.doi.org/10.1159/ $\underline{000277635}$

[13] HARTMANN JT, PINTOFFL JP, KRONING H, BOKEMEYER C, HOLTMANN M, et al. Gefitinib in combination with oxaliplatin and 5-fluorouracil in irinotecan-refractory patients with colorectal cancer: a phase I study of the Arbeits gemeinschaft Internistische Onkologie (AIO). Onkologie 2008; 31: 237-241. http://dx.doi.org/10.1159/000122029

[14] SHARMA SP. Cetuximab for metastatic colorectal cancer. Lancet Oncol 2007; 8: 673. http://dx.doi.org/10.1016/S14702045(07)70224-3

[15] JONKER DJ, O`CALLAGHAN CJ, KARAPETIS CS, ZALCBERG JR, TU D, et al. Cetuximab for the treatment of colorectal cancer. N Engl J Med 2007; 357: 2040-2048. http:// dx.doi.org/10.1056/NEJMoa071834

[16] CHEN MC, CHIANG FF, WANG HM. Cetuximab plus chemotherapy as first-line treatment for metastatic colorectal cancer: Effect of KRAS mutation on treatment efficacy in Tai- 
wanese patients. Neoplasma 2013; 60: 561-567. http://dx.doi. org/10.4149/neo $2013 \quad 073$

[17] GOLDBERG RM, VENOOK AP, SCHILSKY RL. Cetuximab in the treatment of colorectal cancer. Clin Adv Hematol Oncol 2004; 2: 1-10; quiz 11-12.

[18] ELEZ E, ALSINA M, TABERNERO J. Panitumumab - an effective long-term treatment for patients with metastatic colorectal cancer and wild-type KRAS status. Cancer Treat Rev 2010; 36 Suppl 1: S15-16. http://dx.doi.org/10.1016/S03057372(10)70003-7

[19] WISINSKI KB, MULCAHY MF, BENSON AB. 3rd Panitumumab in metastatic colorectal cancer. Clin Adv Hematol Oncol 2007; 5: 10-11.

[20] GIUSTI RM, SHASTRI K, PILARO AM, FUCHS C, CORDOBA-RODRIGUEZ R, et al. U.S. Food and Drug Administration approval: panitumumab for epidermal growth factor receptorexpressing metastatic colorectal carcinoma with progression following fluoropyrimidine-, oxaliplatin-, and irinotecancontaining chemotherapy regimens. Clin Cancer Res 2008; 14: 1296-1302. http://dx.doi.org/10.1158/1078-0432.CCR$\underline{07-1354}$

[21] MANO M, HUMBLET Y. Drug Insight: panitumumab, a human EGFR-targeted monoclonal antibody with promising clinical activity in colorectal cancer. Nat Clin Pract Oncol 2008; 5: 415-425. http://dx.doi.org/10.1038/ncponc1136

[22] TOL J, KOOPMAN M, CATS A, RODENBURG CJ, CREEMERS GJ, et al. Chemotherapy, bevacizumab, and cetuximab in metastatic colorectal cancer. N Engl J Med 2009; 360: 563-572. http://dx.doi.org/10.1056/NEJMoa0808268

[23] SUN W. Angiogenesis in metastatic colorectal cancer and the benefits of targeted therapy. J Hematol Oncol 2012; 5: 63. http://dx.doi.org/10.1186/1756-8722-5-63

[24] THAIRU N, KIRIAKIDIS S, DAWSON P, PALEOLOG E Angiogenesis as a therapeutic target in arthritis in 2011: learning the lessons of the colorectal cancer experience. Angiogenesis 2011; 14: 223-234. http://dx.doi.org/10.1007/s10456-0119208-2

[25] MULDER K, SCARFE A, CHUA N, SPRATLIN J. The role of bevacizumab in colorectal cancer: understanding its benefits and limitations. Expert Opin Biol Ther 2011; 11: 405-413. http://dx.doi.org/10.1517/14712598.2011.557657

[26] USAKOVA V, SEVCIKOVA K, USAK J, BARTOSOVA Z, MIKULOVA $\mathrm{M}$, et al. Bevacizumab in combination with chemotherapy in the first-line treatment of metastatic colorectal carcinoma. Neoplasma 2013; 60: 83-91. http://dx.doi. org/10.4149/neo 2013012

[27] INFANTE JR, REID TR, COHN AL, EDENFIELD WJ, CESCON TP, et al. Axitinib and/or bevacizumab with modified FOLFOX-6 as first-line therapy for metastatic colorectal cancer: A randomized phase 2 study. Cancer 2013.

[28] YANG J, WANG LJ, LIU JJ, ZHONG L, ZHENG RL, et al. Structural optimization and structure-activity relationships of N2-(4-(4-Methylpiperazin-1-yl)phenyl)-N8-phenyl-9Hpurine-2,8-diamine derivatives, a new class of reversible kinase inhibitors targeting both EGFR-activating and resistance mutations. J Med Chem 2012; 55: 10685-10699. http://dx.doi. org/10.1021/jm301365e

[29] PAN Y, XU Y, FENG S, LUO S, ZHENG R, et al. SKLB1206, a novel orally available multikinase inhibitor targeting EGFR activating and T790M mutants, ErbB2, ErbB4, and VEGFR2, displays potent antitumor activity both in vitro and in vivo. Mol Cancer Ther 2012; 11: 952-962. http://dx.doi. org/10.1158/1535-7163.MCT-11-0679

[30] HEO DS, PARK JG, HATA K, DAY RM, HERBERMAN RB, et al. Evaluation of Tetrazolium-based Semiautomatic Colorimetric Assay for Measurement of Human Antitumor Cytotoxicity. Cancer Res 1990; 50: 3681-3690.

[31] CAO ZX, LIU JJ, ZHENG RL, YANG J, ZHONG L, et al. SKLB1028, a novel oral multikinase inhibitor of EGFR, FLT3 and Abl, displays exceptional activity in models of FLT3driven AML and considerable potency in models of CML harboring Abl mutants. Leukemia 2012; 26: 1892-1895. http://dx.doi.org/10.1038/leu.2012.67

[32] ZHANG S, CAO ZX, TIAN HW, SHEN GB, MA YP, et al. SKLB1002, a novel potent inhibitor of VEGF receptor 2 signaling, inhibits angiogenesis and tumor growth in vivo. Clin Cancer Res 2011; 17: 4439-4450. http://dx.doi.org/10.1158/ 1078-0432.CCR-10-3109

[33] ZHOU JP, CHEN X, FENG S, LUO SD, PAN YL, et al. Systems biology modeling reveals a possible mechanism of the tumor cell death upon oncogene inactivation in EGFR addicted cancers. PLoS One 2011; 6: e28930. http://dx.doi.org/10.1371/ journal.pone.0028930

[34] APATI A, JANOSSY J, BROZIK A, BAUER PI, MAGOCSI $\mathrm{M}$ Calcium induces cell survival and proliferation through the activation of the MAPK pathway in a human hormonedependent leukemia cell line, TF-1. J Biol Chem 2003; 278: 9235-9243. http://dx.doi.org/10.1074/jbc.M205528200

[35] SHAHEEN RM, AHMAD SA, LIU W, REINMUTH N, JUNG $\mathrm{YD}$, et al. Inhibited growth of colon cancer carcinomatosis by antibodies to vascular endothelial and epidermal growth factor receptors. Br J Cancer 2001; 85: 584-589. http://dx.doi. org/10.1054/bjoc.2001.1936

[36] TONRA JR, DEEVI DS, CORCORAN E, LI H, WANG S, et al. Synergistic antitumor effects of combined epidermal growth factor receptor and vascular endothelial growth factor receptor-2 targeted therapy. Clin Cancer Res 2006; 12: 2197-2207. http://dx.doi.org/10.1158/1078-0432.CCR-05-1682

[37] BROWN DC, COLE D, GATTER KC, MASON DY Carcinoma of the cervix uteri: an assessment of tumour proliferation using the monoclonal antibody Ki67. Br J Cancer 1988; 57: 178-181. http://dx.doi.org/10.1038/bjc.1988.37

[38] PARAST CV, MROCZKOWSKI B, PINKO C, MISIALEK S, KHAMBATTA G, et al. Characterization and kinetic mechanism of catalytic domain of human vascular endothelial growth factor receptor-2 tyrosine kinase (VEGFR2 TK), a key enzyme in angiogenesis. Biochemistry 1998; 37: 16788-16801. http://dx.doi.org/10.1021/bi981291f 